\title{
Inhibition of ULK2 Autophagic Activity by Its Association with YAP
}

\author{
Sung Hwa Shin \\ Dept. of Biology Education, Chungbuk National University \\ 1, Chundaeg-ro, Seowon-gu, Cheongju, Chungbuk, 28644, Republic of Korea \\ Tel: +82 $432613278 \quad$ E-mail: sunghwashin@gmail.com
}

Eun Jeoung Lee

Dept. of Internal Medicine, Chungbuk National University

1, Chundaeg-ro, Seowon-gu, Cheongju, Chungbuk, 28644, Republic of Korea

Tel: +82432613278Ｅ-mail: babbo89@hanmail.net

\section{Sunghee Hyun}

Dept. Biomedical Laboratory Science, School of Medicin, Eulji University

77 Gyeryong-ro 771 beon-gil, Jung-gu, Daejeon 34824, Korea

Tel: +82-42-259-1751 E-mail: hyunsh@eulji.ac.kr

Dowonkyoung Park

Dept. of Biology Education, Chungbuk National University

1, Chundaeg-ro, Seowon-gu, Cheongju, Chungbuk, 28644, Republic of Korea

Tel: +82 $432613278 \quad$ E-mail: dowonkyoung@gmail.com

\section{Sang Sun Kang(Corresponding author)}

Dept. of Biology Education, Chungbuk National University

1, Chundaeg-ro, Seowon-gu, Cheongju, Chungbuk, 28644, Republic of Korea

Tel: +82 $432613278 \quad$ E-mail: jin95324@ cbu.ac.kr

Received: October 26, 2016 Accepted: November 20, 2016 Published: December 25, 2016 


\section{Abstract}

Uncoordinated 51-like kinase 2 (ULK2) is a member of the serine/threonine kinase family that functions an essential role regulating autophagy in mammalian cells. As autophagy is implicated in normal cellular homeostasis and multiple diseases, better mechanistic insight will drive the development of novel therapeutic approaches. Here, we present evidence that ULK2 interacts with YAP for its degradation and subcellular localization. A potential PPXY motif $\left({ }^{328} \mathrm{PPnY}^{331}\right)$ was identified, which is similar with the consensus PPxY motif in ULK2 S/P domain. The P329A (PA) mutation in the PY motif of ULK2 abolished the YAP-ULK2 association. At first, we observed that ULK2 physically interacted to YAP in vivo and in vitro, using a pull-down approach. Secondly, ULK2 and YAP co- localized at the apical (or tight junction) membrane as visualized by confocal microscopy. Furthermore, the PA mutant substantially increased during autophagy than that of wild-type ULK2 or the P242A mutant in transient transfection assays. Thus, the association between ULK2 and YAP through the WW domain links autophagy and the Hippo signal transduction pathway.

Keywords: Autophagy, Hippo signal transduction, protein-protein interaction: protein subcellular localization, ULK2, WW domain, YAP

\section{Introduction}

Autophagy plays a role in normal cellular homeostasis and multiple diseases; thus, better mechanistic insight will drive development of novel therapeutic approaches (Codogno, Ogier-Denis, \& Houri, 1997; Galliot, 2006; Kelekar, 2005; Terman \& Brunk, 2005). Similar to other ULK family members, uncoordinated 51-like kinase 2 (ULK2) also plays a central role in the autophagy signaling pathway (McAlpine, Williamson, Tooze, \& Chan, 2013; Yan et al., 1999). ULK2 is also present ubiquitously, like ULK1, and its function is overlapped with that of ULK1. Because ULK2 compensates for deletion of ULK1, the specific roles of ULK2 or ULK1 in autophagy are unclear (McAlpine et al., 2013; Yan et al., 1999; Zhou et al., 2007). ULK2 may also be involved in determining cell fate, metabolism, transcriptional control, and oncogenesis (Y. Chen et al., 2014; Dodson, Darley-Usmar, \& Zhang, 2013). Other Studies have suggested that ULK2 activity must be carefully regulated by mechanisms that are individually tailored for each substrate to avoid indiscriminate phosphorylation by ULK2. Although the mechanisms regulating ULK2 during autophagy are not fully understood, precise regulation appears to be achieved through a combination of phosphorylation, localization, and interactions with ULK2 binding proteins (Chan, Longatti, McKnight, \& Tooze, 2009; Jung et al., 2009). However, unlike ULK1, which is found primarily in the cytoplasmic region, ULK2 is located predominantly in nuclei but is also observed in the cytosol and mitochondria (Egan et al., 2011; Y. Huang, Chuang, \& Ratovitski, 2011).

Yes associated protein (YAP) and transcriptional co-activator with a PDZ-binding motif (TAZ) are both localized in the cytoplasm and nucleus where they regulate gene transcription (Andre \& Springael, 1994; H. I. Chen \& Sudol, 1995; Kanai et al., 2000; Sudol, Bork, et al., 1995; Sudol, Chen, Bougeret, Einbond, \& Bork, 1995). Thus, nuclear accumulation of YAP/TAZ is a key determinant of their functions. Phosphorylation by linker for large tumor suppressor (LATS) proteins is the main input for YAP/TAZ subcellular localization, leading 
to sequestration of YAP/TAZ in the cytoplasm. Phosphorylation of YAP S127 creates a binding consensus for 14-3-3 proteins that contribute to maintain YAP/TAZ in the cytoplasm (Basu, Totty, Irwin, Sudol, \& Downward, 2003; J. Huang, Wu, Barrera, Matthews, \& Pan, 2005; Kanai et al., 2000; Vassilev, Kaneko, Shu, Zhao, \& DePamphilis, 2001; Wang, Degerny, $\mathrm{Xu}$, \& Yang, 2009).

YAP/TAZ contains a WW domain composed of 38 amino acids, making it among the smallest globular domains known to mediate protein-protein interactions. These WW domains interact with the core PPxY motif sequence for Hippo signal transduction. Many structural and biochemical studies on YAP have revealed that this shuttle protein binds many proteins containing the PPxY motif and its WW domain (Kanai et al., 2000; Sudol, Bork, et al., 1995; Sudol, Chen, et al., 1995).

ULK2 is considered a YAP WW domain interacting protein based on WW domain binding motif consensus amino acid sequence information (Yan et al., 1999). Therefore, in this study, we show that ULK2 is a YAP target protein through interactions between the WW domain and the $\left({ }^{328} \mathrm{PPnY}^{331}\right)$ motif. In addition, we provide evidence that YAP interactions promote apical (or tight junction) membrane or cytoplasmic localization of ULK2, leading to downregulation of ULK2 autophagic activity. Thus, our results provide insight into the molecular mechanisms underlying the role of ULK2 during cross-talk between autophagy and the Hippo signaling pathway.

\section{Materials and Methods}

\subsection{Reagents}

The protease inhibitor cocktail was obtained from Roche Molecular Biochemical (Mannheim, Germany) in tablet form. Monoclonal and polyclonal antibodies against YAP, ULK2, and WIPI were purchased from Abcam (Cambridge, UK) or Cell Signaling Technology (Boston, MA, USA).

\subsection{Cell Culture and Transfections}

HEK293 or NIH 3 T3 cells were cultured in DMEM medium supplemented with $10 \%$ (v/v) heat-inactivated fetal bovine serum (FBS) and 1,000 U penicillin-streptomycin (Gibco BRL, Grand Island, NY, USA). The cells were maintained in DMEM supplemented with $10 \%(\mathrm{v} / \mathrm{v})$ FBS and antibiotics. Transfections were conducted with Lipofectamine and Plus reagents (Invitrogen, Carlsbad, CA, USA) in accordance with the manufacturer's instructions.

\subsection{Plasmid Constructs}

Human ULK2 cDNA was obtained from the Korea Human Gene Bank (Gene ID; KIAA0623) and cloned in the pEGFP2 vector (Clontech, Palo Alto, CA, USA; enhanced green fluorescent protein [EGFP] was added to the ULK2 $\mathrm{N}$-terminus). To generate the P329A (PA) mutant, ULK2 was subjected to site-directed mutagenesis using this following mutagenic primers: forward 5'-CCA CCA TTG GGT CCT GCC AAC TAT CTA CAA GTT-3' and reverse 5'-TTG TAG ATA GTT GGC AGG ACC CAA TGG TGG GGA-3'; for the P242A mutant: forward, 5'-CCC AGA GAA ACA TCA GCT TAT TTG GCT AAT CTC-3' 
and reverse 5'-ATT AGC CAA ATA AGC TGA TGT TGT TTC TCT GGG AAT-3') were used with the QuikChange Multi Mutagenesis Kit (Stratagene, West Cedar, TX, USA) according to the manufacturer's instructions.

\subsection{Immunoprecipitation}

Cells were routinely analyzed 48 hours post-transfection. The cells were rinsed with ice-cold phosphate-buffered saline and resuspended in $1 \mathrm{~mL}$ extraction buffer $[10 \mathrm{mM}$ Tris- $\mathrm{HCl} \mathrm{pH} 7.4$, $1 \mathrm{mM}$ EDTA, $5 \mathrm{mM}$ DTT, $100 \mathrm{mM} \mathrm{NaCl}, 1.0 \%$ Triton X-100, $60 \mathrm{mM}$-octylglucoside, 1 $\mathrm{mM}$ vanadate, $100 \mu \mathrm{M}$ molybdate, $20 \mathrm{mM}$ sodium fluoride, and protease inhibitor cocktail of 1 tablet/10 $\mathrm{ml}$ extraction buffer). The pre-cleaned lysate was incubated for 1 hour at $4{ }^{\circ} \mathrm{C}$ with the appropriate antibody, and the resulting immune complexes were collected on Protein A-Sepharose beads (Pharmacia Co., Piscataway, NJ, USA). Immune complexes were captured by centrifugation, washed extensively in lysis buffer, and solubilized with $2 \times$ sample buffer prior to $10 \%$ sodium dodecyl sulfate-polyacrylamide gel electrophoresis (SDS-PAGE).

\subsection{ULK2 or YAP Pull-down Assay}

Whole cell lysates of HEK293 cells transiently expressing ULK2 were pre-cleaned with glutathione agarose beads, then $1 \mu \mathrm{g}$ each of the glutathione agarose-tagged recombinant ULK2 or YAP preparations was added to separate samples followed by a $2 \mathrm{hr}$ incubation at $4^{\circ} \mathrm{C}$ on an end-over-end rotating shaker to allow for the association between ULK2 and YAP. Associated protein complexes were collected using a slurry of glutathione agarose beads and washed extensively. After resuspension in $2 \times$ Laemmli sample buffer, the samples were analyzed by $10 \%$ SDS-PAGE, and western blotting was performed with YAP or ULK2 antibodies.

\subsection{Immunoblotting}

Pulled-down or immunoprecipitated ULK2 (or YAP) was resolved by 10\% SDS-PAGE and transferred to a nitrocellulose membrane. Membranes were incubated in blocking buffer $(5 \%$ dried skim milk in PBS and $0.05 \%$ Tween-20) and probed with specific antibodies, followed by a horseradish peroxidase-conjugated secondary antibody. Immune complexes were detected using a commercial Western blotting detection system (Pierce, Rockford, IL, USA).

\subsection{Confocal Microscopy}

HEK293 cells were seeded overnight at $60 \%$ confluence onto culture slides coated with human fibronectin (SPL, Seoul, Korea). The following day, the cells were transfected with the ULK2/EGFP construct and allowed to grow for an additional $48 \mathrm{~h}$. Cells were washed several times with ice-cold PBS and fixed in $2 \%$ paraformaldehyde for $10 \mathrm{~min}$. The fixed cells were permeabilized with $0.1 \%$ Triton X-100 for 10 min and blocked for 2 hours in PBS containing 5\%bovine serum albumin (Aurion, Best, The Netherlands) and $0.1 \%$ Tween. Following incubation with a polyclonal antibody against YAP, the cells were washed and stained further with rabbit IgG against LC3 II or WIPI (Bio-Protocol, Palo Alto, CA, USA) or conjugated donkey anti-rabbit $\mathrm{IgG}$ prior to processing the slides for immunofluorescence. After a 20 -min incubation at $37^{\circ} \mathrm{C}$, the cells were fixed, permeabilized, and anti-YAP or 


\section{Mll Macrothink}

Journal of Biology and Life Science ISSN 2157-6076 2017, Vol. 8, No. 1

ULK2 antibody was added. Alexa Fluor 568 or 488-conjugated donkey anti-rabbit or anti-mouse (Molecular Probes, Inc., Eugene, OR, USA) was used as a secondary antibody. The confocal laser scanning microscopy (CLSM) analysis was performed using a LSM710 (Zeiss, Zena, Germany) at the Center for Research Instruments and Experimental Facilities, Chungbuk National University. Using Profile in the ZEN program, which was provided by the manufacturer, protein co-localization was observed and the confocal microscopic photographs were scanned.

\subsection{Quantitative Co-localization}

The co-localization analysis was performed on digitized CLSM images using ZEN2012 software (ZEISS, Germany), where a defined ULK2 threshold signal above the background level (secondary antibody alone control) was overlaid onto that of the IMP signal to produce a merged image to colocalized the ULK2 and YAP pixels. The pixel intensity of the merged image was expressed as a percentage of the total YAP signal to calculate the mean percentage of YAP colocalized with ULK2 (Barlow, Macleod, Noppen, Sanderson, \& Guerin, 2010; Lieu et al., 2014). Pearson's correlation coefficient (PCC) of the co-localization between Yap and ULK2 was measured with an LSM710 microscope.

\subsection{FACS Analysis}

Cells were transfected with ULK2 (wild-type [WT]), the PA mutant, the enhanced green fluorescent protein (EGFP) vector, and the rate of apoptosis was measured using the Annexin V-PE apoptosis detection kit (BD Biosciences, Piscataway, NJ, USA), according to the manufacturer's instructions. Cells were vortexed gently and incubated for $15 \mathrm{~min}$ at $25^{\circ} \mathrm{C}$ in the dark. Binding buffer $(400 \mu \mathrm{l})$ was added to each tube. Fluorescence-activated cell sorting (FACS) was performed within $1 \mathrm{~h}$ using a FACS Calibur (BD BioSciences) at the Core Facility of Chungbuk National University.

\section{Results}

\subsection{Endogenous ULK2 Interacts with YAP and Localizes in the Apical Region (Tight Junction) of the Membrane}

ULK2 contained the $\left({ }^{328} \mathrm{PPnY}^{331}\right.$ ) motif within its S/P space domain (Figure 1A) (Yan et al., 1999). The presence of this motif in ULK2 suggests that ULK2 can bind the YAP65 (YAP) WW1 domain (H. I. Chen \& Sudol, 1995; Sudol, Chen, et al., 1995). Therefore, we determined whether endogenous YAP formed a protein complex with ULK2 in HEK293 cells. As shown in Figure 1B, the ULK2 immunoprecipitate contained YAP. Antibodies directed against YAP successfully captured ULK2 from the same lysates, corroborating the hypothesis that the two proteins associate physically. Furthermore, we examined whether ULK2 is associated with YAP in cells by CLSM. Endogenous ULK2 (green) and YAP (red) co-localized in the nucleus and cytoplasm of HEK293 cells (yellow) (Figure 1C). ULK2 and YAP (white spot) co-localized in the enlarged picture of the specific merged region in Figure 1C. Endogenous ULK2 was also found with YAP primarily on the membrane apical region (or tight junction) of 3T3, MDCK2, CHO and HepG2 cells (data not shown). PCC of the co-localization between Yap and ULK2 was provided in the right side (Barlow et al., 2010; 
Lieu et al., 2014). PCC between YAP and ULK2 was measured as $0.69 \pm 0.12(\mathrm{n}=10)$. From these results, we strongly suggest that endogenous ULK2 interacts with YAP in the cells, and that ULK2 is localized in the membrane (or tight junction) asymmetrically.

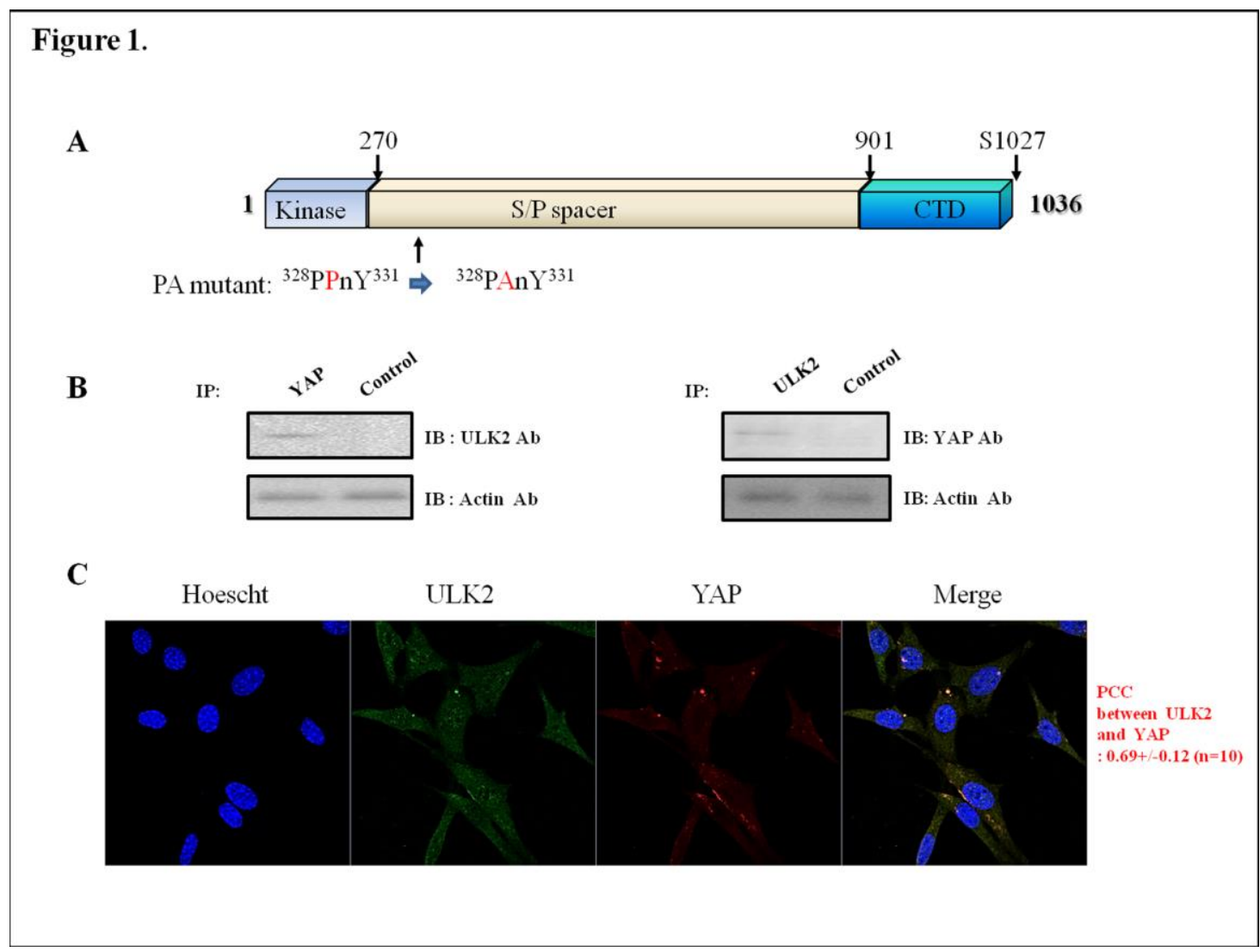

Figure 1. Interaction between ULK2 and YAP and apical (tight junction) membrane or cytoplasmic localization of both ULK2 and YAP

(A) ULK2 (Gene ID: KIAA0623) contains a putative-conserved YAP binding motif: $\left({ }^{328} \mathrm{PPnY}^{331}\right)$ within the serine/proline (S/P)-rich space domain. The PA point mutant (PA) was prepared to define the binding motif. Mutated sequences (PA mutant: ${ }^{328} \mathrm{PAnY}^{331}$ ) are indicated with arrows. The PA mutant $\left({ }^{328} \mathrm{PAnY}^{331}\right)$ was constructed. Regions of the protein in the C-terminal domain (CTD), which that are involved in membrane attachment and interaction with Atg13-focal adhesion kinase family-interacting protein 200 (FIP200), are indicated. (B) An immunoblot (IB) analysis was performed using antibody against YAP (left) following immunoprecipitation (IP) with anti-ULK2 antibody. Anti-YAP immunoprecipitated complexes were subjected to IB analysis using anti-ULK2 antibody (right). Co-IP of YAP with ULK2 confirmed the presence of the ULK2-YAP complex in the cell. An unrelated antibody was used as a negative IP control. An actin antibody was used as a control for the IB analysis (bottom). (C) Confocal fluorescence micrographs showing endogenous ULK2 and YAP in HEK293 cells. These proteins were visualized by immunofluorescence in fixed and permeabilized cells using monoclonal or polyclonal antibodies against human YAP or ULK2 and Alexa Fluor 568 conjugated donkey anti-rabbit IgG or Alexa Fluor 488 conjugated mouse anti-rabbit IgG. The yellow pattern resulted from merging red and green and indicates co-localization of the proteins, mainly in the nuclear membrane. The nuclear region was visualized with Hoescht staining (blue). An enlarged co-localization picture of the specific merged region is shown below (white color) to show ULK2 and YAP co-localization. Pearson's correlation coefficient (PCC) for the co-localization between Yap and ULK2 is provided on the right $(0.69 \pm$ $0.12 ; \mathrm{n}=10)$. 


\section{Macrothink}

\subsection{The ULK2 $\left({ }^{328} P P n Y^{331}\right)$ Motif is Responsible for Binding with the YAP WW Domain}

As shown in Figure 1B and C, ULK2 interacted with YAP in vitro. Because ULK2 contains the well-conserved YAP binding motif in its S/P spacer (Figure 1A), we assumed initially that $\left({ }^{328} \mathrm{PPnY}^{331}\right)$ was the ULK2 PY motif (H. I. Chen \& Sudol, 1995; Sudol, Chen, et al., 1995). A co-immunoprecipitation experiment was conducted to demonstrate the interaction between ULK2 and YAP through its putative PY-motif (Figure 2A). EGFP-ULK2 WT, the PA point mutant $\left({ }^{328} \mathrm{PPnY}^{331} \rightarrow{ }^{328} \mathrm{PAnY}^{331}\right)$, and P242A was transfected into HEK293 cells. The mutant P242A was used as the negative mutational control. After $48 \mathrm{~h}$, the cells were lysed, and the immunoprecipitation was conducted with rabbit anti-EGFP antibody. To examine whether the immunoprecipitant brought down YAP together, the western blot assays were performed with mouse anti- YAP, anti-ULK2 or actin antibodies (1: 1000 dilution). As shown in Figure 2B, EGFP-ULK2 WT and P242A was able to specifically bring down the endogenous YAP, while PA failed to interact YAP. This result, therefore, suggested that $\left({ }^{328} \mathrm{PPnY}^{331}\right)$ motif of ULK2 S/P spacer is responsible as PY-motif to bind WW domain of YAP.

Figure 2.

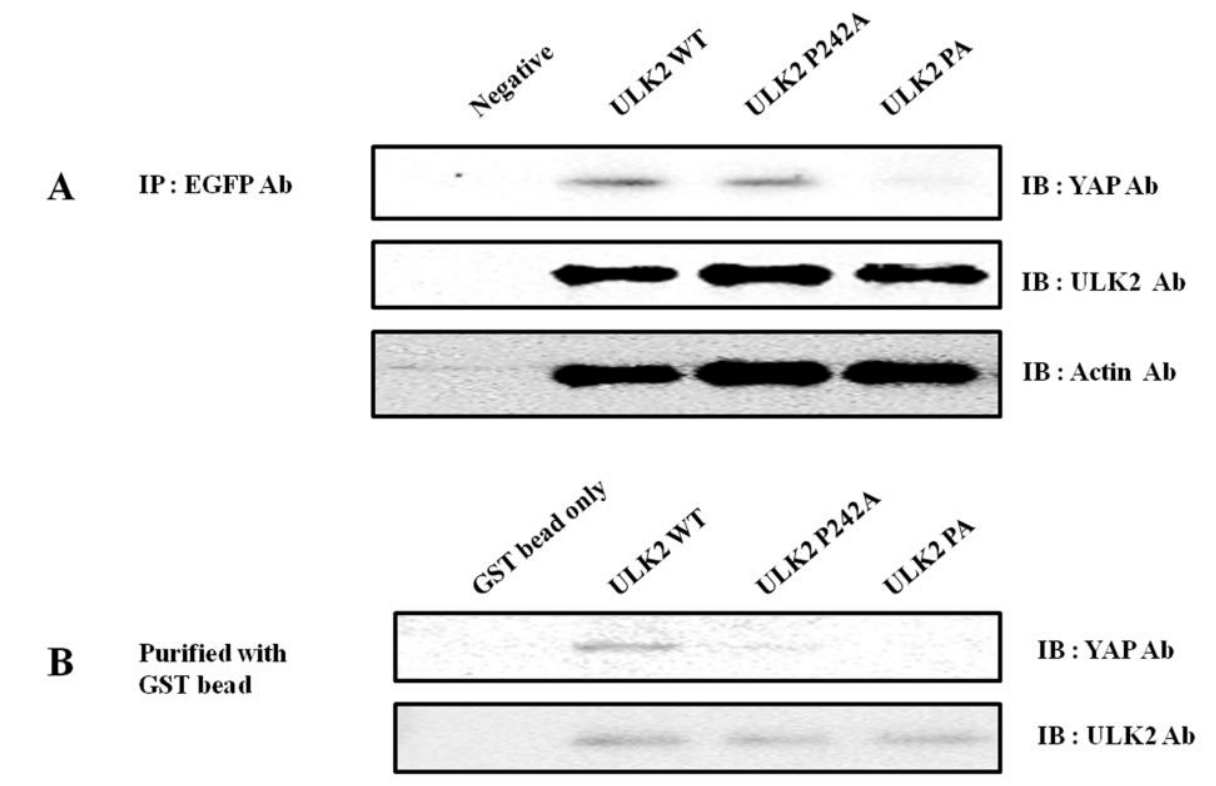

Figure 2. The ULK2 $\left({ }^{328} \mathrm{PPnY} \mathrm{Y}^{331}\right)$ motif is responsible for the interaction between ULK2 and YAP

(A) Co-immunoprecipitation of ULK2 WT, P242A, or PA with YAP. HEK293 cells were transiently transfected with WT EGFP-ULK2 or the PA plasmid. The cells were lysed after $48 \mathrm{~h}$, and a Western blot was conducted with anti-EGFP antibody and protein A agarose beads. Western blot assays were performed with rabbit anti-ULK2 or mouse anti-YAP antibodies. The YAP band $(\sim 66 \mathrm{kDa})$ is shown. A Western blot was also performed with anti-actin antibody (bottom) to monitor protein quantity in the cell lysate. (B) Pull-down 
analysis of YAP with GST fusion ULK2 WT, P242A, or PA mutants. GST-fusion proteins encompassing the C-terminal ULK2 C-domain were constructed and expressed in E. coli. Approximately $0.1 \mathrm{mg}$ of the WT, P242A, or PA fusion proteins bound to glutathione-Sepharose beads (bottom lane) were incubated with HEK 293 cell lysates. Western blot assays were performed with rabbit anti-ULK2 or mouse anti- YAP antibodies.

To gain more evidences for the interaction between ULK2 and YAP through its putative PY-motif, the pull-down experiment with GST-ULK2 protein purified in E. coli was performed (Figure 2B). GST-ULK2 WT or GST-ULK2 mutant (P242A or PA) was purified with the glutathione bead from E. coli lysate. A pull-down assay in the cell lysate was conducted with GST-ULK2 beads. The purified GST-ULK2 fusion protein was incubated with the same amount of HEK293 total cell lysate (equivalent to a 10mm confluent culture) Western blot assays were performed with rabbit anti-EGFP or mouse anti- YAP antibodies. As shown in Figure 2B, both GST-ULK2 WT and the P242A mutant specifically brought down the endogenous YAP in HEK293 cell. However, GST-ULK2 PA fusion protein failed to interact with YAP (Figure $2 \mathrm{~B}$ ). Taken together, these results indicate that the ${ }^{328} \mathrm{PPnY}{ }^{331}$ motif is crucial for the interaction between ULK2 and YAP.

\subsection{Interaction between Exogenous ULK2 and YAP Is Required for Asymmetrical Membrane (or Tight Junction) Localization of ULK2}

CLSM was performed to better understand the effects of the interactions between ULK2 and YAP (Figure 3). We investigated whether the putative ULK2 $\left({ }^{328} \mathrm{PPnY}^{331}\right)$ motif could direct the interaction of this protein with the YAP WW domain. Similar to the endogenous ULK2 result shown in Figure 1D, exogenous WT EGFP-ULK2 (green) and YAP (red) were asymmetrically co-localized at tight junctions of the plasma membrane (yellow) (Figure 3A). However, the exogenous EGFP-ULK2 PA mutant (green) did not co-localize with YAP (red) (Figure 3B), which was probably due to the mutation in the YAP binding site. The EGFP-ULK2 PA mutant (green) was localized to the nucleus, rather than to the membrane (Figure 3B). However, the exogenous EGFP-ULK2 P242A mutant (green) co-localized with YAP (red) in the asymmetrical (tight junction) membrane (Figure 3C), likely due to its YAP binding site. Taken together, these data strongly suggest that YAP interacts with ULK2 through the $\left({ }^{328} \mathrm{PPnY}^{331}\right)$ consensus motif of the latter protein in the S/P space domain (Figure 1A), and that protein-protein interactions between ULK2 and YAP are required for asymmetrical (or tight junction) membrane localization of ULK2 (Figure 3B). PCC between the PA mutant and YAP was $0.36 \pm 0.06 ; n=10)$, which was half of that of ULK WT $(0.65 \pm$ $0.05 ; \mathrm{n}=10)$ or the P242A mutant $(0.58 \pm 0.08 ; \mathrm{n}=10)$. Therefore, these observations suggest that the interaction between ULK2 and YAP through its WW domain binding motif $\left({ }^{328} \mathrm{PPnY}^{331}\right)$ is required for the asymmetrical localization of ULK2 in the membrane (or tight junction). 


\section{Macrothink}

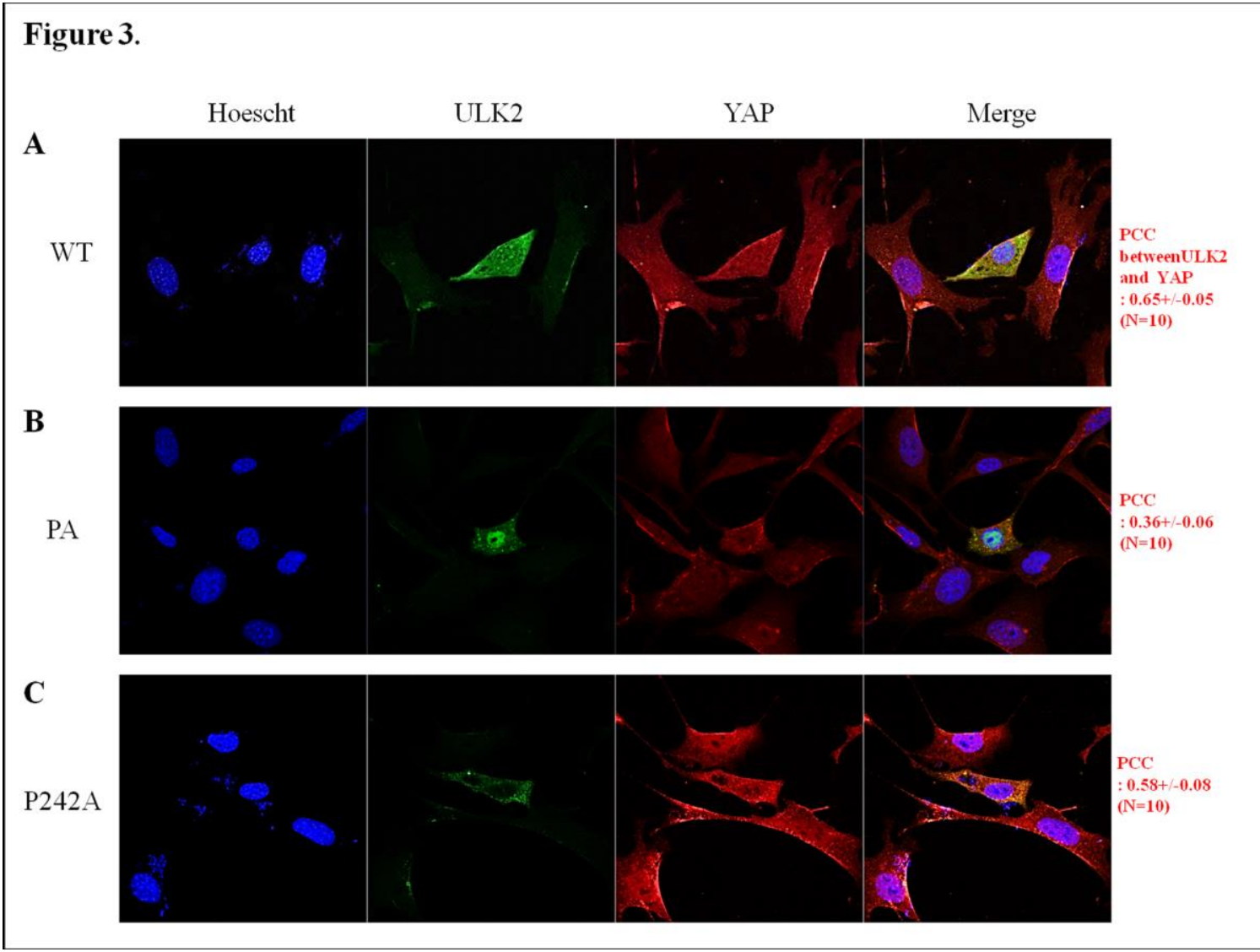

Figure 3. Subcellular localization of exogenous WT ULK2, PA, or the P242A mutant

Confocal fluorescence micrographs of WT EGFP-ULK2, PA, or P242A in HEK293 cells. All EGFP constructs fluoresced green, whereas YAP fluoresced red. Transfected EGFP- WT ULK2 merged with YAP dominantly in the nucleus (A). Yellow resulted from merging red and green and indicates co-localization of the proteins, mainly in the nucleus, similar to the results obtained for endogenous ULK2 shown in Figure 1C. The transfected EGFP-ULK2 PA mutant was detected in the nucleus and did not co-localize with YAP in the cytoplasm (B), Even though YAP (red color) is localized asymmetrically, the EGFP-ULK2 PA mutant is distributed evenly. The P242A mutant was used as the negative control (C). The P242A mutant was co-localized with endogenous YAP asymmetrically. Cell nuclear region was visualized with Hoescht staining (blue). Representative images based on five replicate experiments for each construct are shown. Pearson's correlation coefficient (PCC) for the co-localization between Yap and ULK2 is shown at right. The PA mutant was neither co-localized with YAP nor asymmetrically.

\subsection{The ULK2 PA Mutant Enhances Its Autophagic Activity}

From the unexpected observation that the cytoplasmic ULK2 PA mutant facilitates the appearance of a greater number of autophagosomes (or puncta) than that of nuclear-localized ULK2 (Figure 3B), we assumed that the cytoplasmic ULK2 PA mutant exerts more autophagic activity than ULK2 WT does. To test this hypothesis, we conducted another CLSM analysis of the endogenous human WD-repeat protein interacting with phosphoinositides (WIPI; another autophagy marker protein) and each ULK2 construct (Figure 4A C) $12 \mathrm{~h}$ after inducing starvation (Proikas-Cezanne, Ruckerbauer, Stierhof, Berg, 


\section{Ml Macrothink}

Journal of Biology and Life Science ISSN 2157-6076 2017, Vol. 8, No. 1

\& Nordheim, 2007; Proikas-Cezanne et al., 2004). WIPI also mainly co-localized with the mutant protein in the cytoplasm in cells transfected with ULK2 PA (Figure 4B), compared with WIPI in ULK2 WT- (Figure 4A) or P242A-mutant (Figure 4C) transfected cells. PCC of the co-localization between ULK2 and WIPI was provided on the right side (Barlow et al., 2010; Lieu et al., 2014). PCC between the PA mutant and WIPI was $0.90 \pm 0.04 ; n=10$ ), which was twice than that of ULK WT $(0.56 \pm 0.08 ; \mathrm{n}=10)$ or the P242A mutant $(0.59 \pm$ $0.05 ; \mathrm{n}=10$ ). Thus, our observations suggest that ULK2 interacts with WIPI in the cells, and that ULK2 PA mutant (which does not associate with YAP) facilitates more autophagy than that of ULK2 WT or P242A mutant (as a negative control).

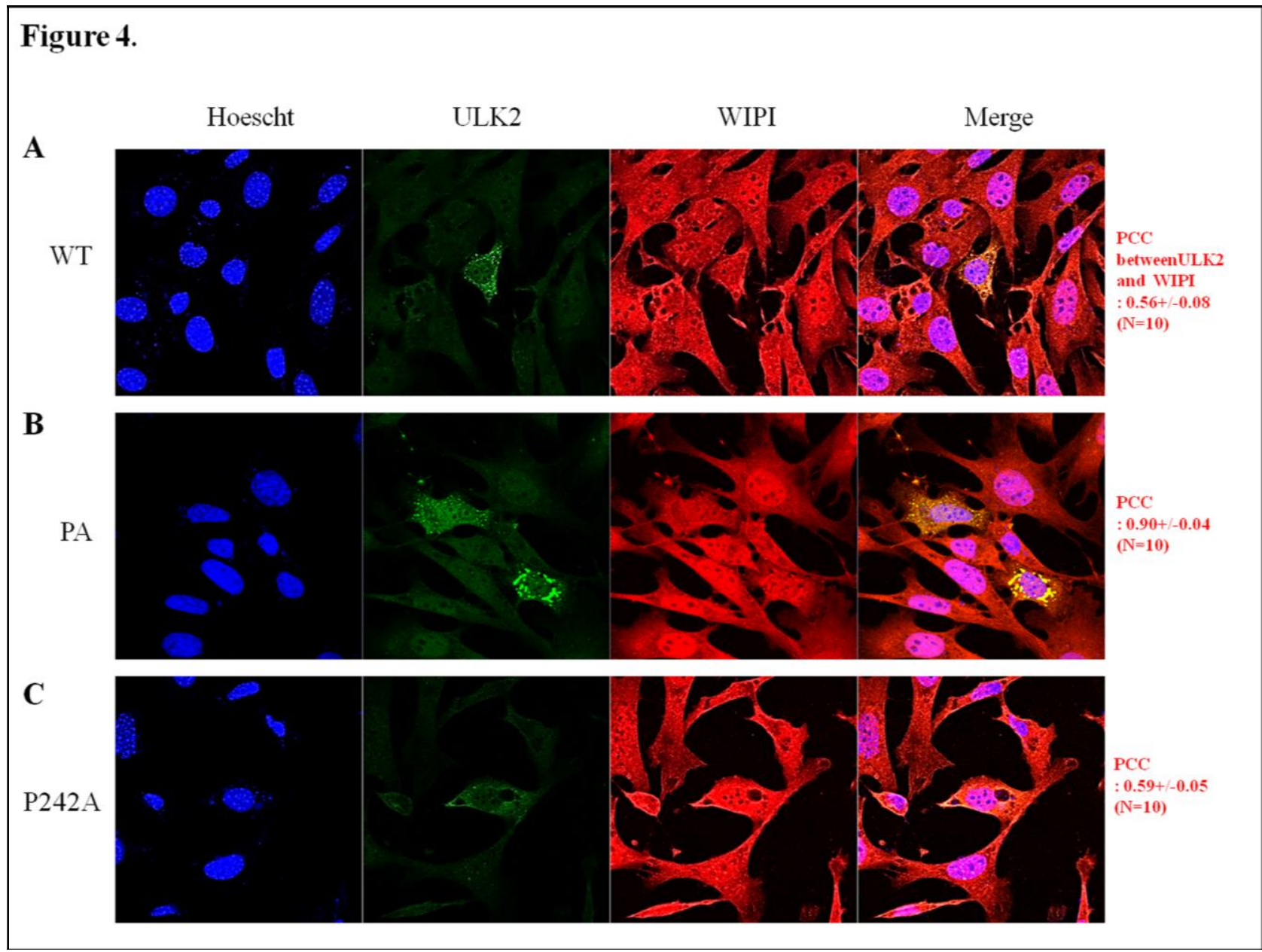

Figure 4. Comparison between the autophagic ability of WT, ULK2 PA, or the P242A mutant

WT EGFP ULK2 or its PA, and P242A mutant were transfected into HEK29 cells. The cells were stained with the endogenous human WD-repeat protein interacting with phosphoinositides (WIPI; another autophagy marker protein) antibody $12 \mathrm{~h}$ after inducing starvation to visualize co-localization of WT ULK2 (A), PA (B), or P242A (C) in HEK293 cells. Confocal fluorescence micrographs were taken as described in the Figure 3 legend. An antibody against WIPI was used per the manufacturer's recommendations. The number of autophagosomes (or puncta) was counted as a measure of autophagic activity. Pearson's correlation coefficient (PCC) for co-localization between Yap and ULK2 is indicated at right $(\mathrm{n}=10)$. The PCC value between WIPI and PA was 1.5 times that between WIPI and WT ULK2. 


\subsection{PA Mutational Effect of ULK2 on Cell Viability}

We measured cell viability using FACS analysis to determine whether subcellular localization of ULK2 influenced cell viability. As shown in Table 1, the FACS results indicated that cells containing ULK2 PA (reduced YAP binding and enhanced autophagy activity; Figures 3 and 4) had higher apoptosis rates than cells transfected with WT EGFP-ULK2, PA, P242A, or the EGFP vector alone. Instead, the promotion of ULK2 nuclear localization by the PA mutation (less binding with YAP) enhanced both its autophagic activity and programmed cell death in our system (Table 1 and Figure 4). Therefore, although it is possible that the ULK2 PA property changed, compared with that of ULK2 WT, from the point mutation, our observations suggest that subcellular localization (or autophagy activity) of ULK2 was related with the YAP interaction. However, it is unclear whether the protein-protein interaction between YAP and ULK2 depends on the cell cycle.

Table 1. Comparison of the effect of WT ULK2 and its PA mutant on cell survival

\begin{tabular}{ll}
\hline ULK2 & Rate of apoptosis (\%) by FACS \\
\hline WT & $24 \pm 3$ \\
PA mutant (P329A) & $38 \pm 4$ \\
Control (P242A) & $23 \pm 4$ \\
(Vector only) & $7 \pm 2$ \\
Mean values of 10 replicates & \\
\hline
\end{tabular}

Cells were transfected with WT EGFP-ULK2 or its PA or EGFP vector, and the apoptosis rate was measured by FACS. The EGFP-ULK2 PA mutant was localized in the cytoplasm and promoted cell apoptosis significantly more than that of WT ULK2, which was localized in the tight junction; the apoptotic effect of the ULK2 PA mutant was roughly 1.5 times that of ULK2 WT. The apoptotic effect of WT or PA was correlated WT or PA with WIPI (Figure 4).

In summary, our data clearly show that ULK2 binds to the YAP WW domain via the $\left({ }^{328} \mathrm{PPnY}^{331}\right)$ motif in the S/P space (Figure 1), resulting in asymmetrical membrane (or tight junction) localization (Figures 3 and 4). In addition, the dissociation with YAP (due to the PA mutation) increased autophagy activity (Figure 4), and apoptosis (Table 1). Therefore, the association between ULK2 and YAP through the WW domain seems to provide a link between Hippo signaling and autophagy activity.

\section{Discussion}

The role of ULK1/2 in signal transduction has been most clearly characterized in the context of autophagy kinase signaling, in which ULK1 functions as a hub protein kinase (Codogno et al., 1997; Galliot, 2006; Kelekar, 2005; McAlpine et al., 2013; Terman \& Brunk, 2005; Yan et al., 1999; Zhou et al., 2007). In this study, we describe the in vitro and in vivo interactions between ULK2 (a member of the autophagy kinase family) and YAP that resulted in localization of ULK2 to the tight junction (Figure 1). We demonstrated that ULK2 and YAP are strongly associated using co-immunoprecipitation and CLSM analyses (Figures 1-3). In addition, we presented clear evidence that this interaction is mediated through the ULK2 $\left({ }^{328} \mathrm{PPnY}^{331}\right)$ binding motif to the YAP65 WW1 domain (H. I. Chen \& Sudol, 1995; Sudol, 


\section{MInstitute"}

Chen, et al., 1995).

We demonstrated here that ULK2 functions as a YAP interacting protein, resulting in localization of ULK2 in the tight junction (Figures 1, 3, and 4). Therefore, ULK2 may play a particular role in the Hippo signal pathway, even though this specific role is presently unclear. It also remains to be determined whether both of these motifs contribute to the observed increase in survival of cells containing the ULK2 mutant and the autophagic activity of this protein (Table 1 and Figure 4). Although our observations suggest that the interaction between ULK2 and YAP controls localization of ULK2 to the tight junction (Figures 3 and 5) and reduces ULK2 autophagic activity (Figures 4 and 5), our results also raise the question as to the function of ULK2 in the nucleus because the interaction between ULK2 and YAP reduced ULK2 autophagic activity (Figure 4) and enhanced phosphorylation of its serine residues (Figure 4). In addition, it remains to be determined whether the ULK2 PA mutation itself $\left({ }^{328} \mathrm{PAnY}^{331}\right)$ affects kinase activity, regardless of protein-protein interactions with YAP. In this study, we observed that ULK2 PA was less self-phosphorylated than that of WT ULK2 (Figure 6). Notably, the YAP WW domain binding motif $\left({ }^{328} \mathrm{PPnY}^{331}\right)$ present in ULK2 near its self-phosphorylation site (Figure 1A) is found only in ULK2 among other ULKs, suggesting that ULK2 may have a unique function in autophagy. Previously, we demonstrated that ULK2 was localized in the nucleus rather than the cytoplasm, compared with subcellular localization of ULK1 (which lacks ${ }^{328} \mathrm{PPnY}^{331}$ ) (Figures 1D and 3) (Shin, Lee, Chun, Hyun, \& Kang, 2015). This may explain the difference in autophagic activity between ULK1 and ULK2, which appears to be due to the subcellular localization difference, because the ULK2 PA mutant (mainly localized in the nuclei) also showed different autophagy activity (Figure 4) (Shin et al., 2015). Thus, it is necessary to ascertain whether phosphorylation of ULK2 is necessary to activate and/or regulate the ULK2 subcellular localization by YAP (Figures 3 and 4).

Figure 5.

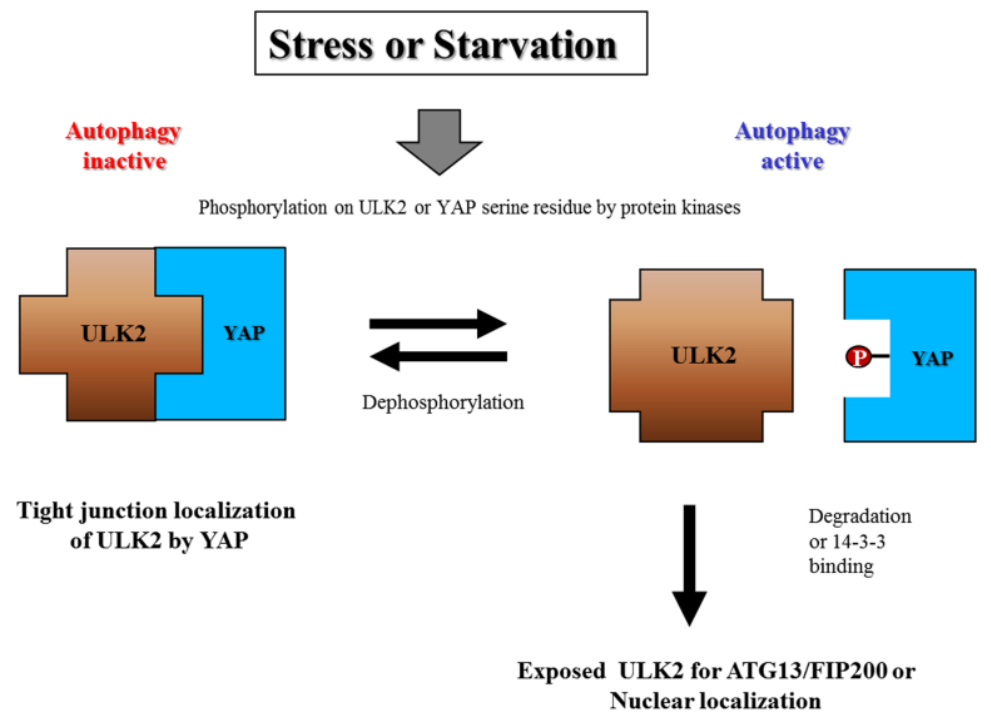

Figure 5. Schematic relationship between ULK2 and YAP 


\section{$\triangle$ Macrothink}

The PA mutant showed more autophagic activity but less serine phosphorylation. Because the mutant predominantly localized in the nucleus and overexpressed YAP2 pulled the endogenous ULK2 into the cytoplasm, we propose that the interaction between ULK2 and YAP2 through WW ligand-domain association plays a role in their subcellular localization. The ULK2 PA mutant easily binds other proteins (such as Atg13 and FIP200) to promote cell autophagy and apoptosis. These protein associations result in hiding of the PY-NLS motif through steric inhibition, resulting in blockade of its nuclear localization by YAP (left). The ULK2 PA mutant, which is free from YAP, is exposed to importins, PA mutant proteins that can be imported into the nucleus effectively (right). The freed ULK2 PA mutant induces cell autophagy or apoptosis. Thus, binding of ULK2 by YAP seems to be cross-talk between autophagy and the Hippo signal pathway.

Although the ULK2 $\left({ }^{328} \mathrm{PAnY}{ }^{331}\right)$ mutant showed a dramatic subcellular difference compared with that of WT, it is unclear whether this affects the protein-protein interactions with Atg13-FIP200 and ULK2 C-terminus (Figure 1A) (Chan et al., 2009; Jung et al., 2009). Other possibilities are not excluded, such as a structural disturbance or other modification changes. PPxY-containing proteins regulate effectors of the Hippo pathway (H. I. Chen \& Sudol, 1995; Sudol, Bork, et al., 1995). WW domain binding motifs also appear to regulate the Hippo pathway at the level of the YAP and TAZ effectors. Several proteins, such as LATS proteins via their PPxY motifs, bind the YAP WW domain leading to YAP phosphorylation, sequestration in the cytoplasm, and inactivation (J. Huang et al., 2005; Sudol, Chen, et al., 1995; Wang et al., 2009). This reduces YAP-induced EMT phenotypes and is associated with reduced tumorigenicity. In fact, the YAP WW domain has a critical role inducing a subset of YAP target genes independent of or in cooperation with TEAD. In addition, mutagenesis of the WW domain diminishes the ability of YAP to stimulate cell proliferation and oncogenic transformation. Two recent studies showed that WW domain-mediated interactions with WBP2 are important for phenotypes induced by Yki47 and TAZ (H. I. Chen \& Sudol, 1995; J. Huang et al., 2005; Sudol, Bork, et al., 1995; Sudol, Chen, et al., 1995; Wang et al., 2009).

We do not know how the association between YAP and ULK2 is related with these functions. YAP contains five putative serine residues that are phosphorylated by ULK2 (our unpublished data). Tyrosine phosphorylation on the PPxY motif has been suggested as a traditional regulation point; however, which tyrosine kinase is responsible is unknown (Figure 1). It is also unknown how binding between the WW domain and its ligand protein is regulated in the cell. Autophagy and the Hippo signal pathway seem to be antagonistic because the association between YAP and ULK2 inhibits their functions. In this study, we demonstrated that autophagic activity (or apoptosis) of the ULK2 PA mutant (which is free from YAP) increased (Figure 5).

The dual functioning Hippo-YAP/TAZ signal pathway is involved in regulating organ size and cancer but autophagy or apoptosis must be connected properly in space and time. Therefore, the association between ULK2 and YAP through the WW domain may be important for signal cross-talk that participates in regulating organ shape and size in a cell context-specific fashion. A transgenic animal with a mutated ULK2 PY motif is required to test this hypothesis.

In conclusion, we demonstrated here that the PY motif $\left({ }^{328} \mathrm{PPnY}^{331}\right)$ in the ULK2 S/P space domain binds to the YAP WW domain. Unlike ULK1, which is localized primarily in the cytoplasm, WT ULK2 is co-localized in the apical (tight junction) membrane through its PY 
motif. Nuclear localization of ULK2 was blocked by association with YAP (Figure 3). In contrast, the ULK2 PA mutant increased its autophagic activity and nuclear localization by dissociating with YAP, which could be a specific drug targeting site. Our observations also suggest Hippo signal transduction cross-talk with autophagy through the protein-protein interactions between ULK2 and the YAP WW1 domain. In addition to the unique presence of PY-NLS in ULK2, the association between ULK2 and YAP through the WW domain seems to be another distinct role from those of ULK1 in autophagy (Shin et al., 2015). Thus, we propose our working hypothesis to explain why cytoplasmic localization of ULK2 through the ${ }^{328} \mathrm{PPnY}^{331}$ motif to the YAP WW domain enhances cell survival (Table 1 and Figure 5).

\section{Acknowledgements}

This study was supported by a Korea Research Foundation grant (NRF-2016R1D1A3B03934269) to S. S. Kang. Shin is a Korean Research fellowship recipient (NRF-2014R1A1A2009622).

\section{References}

Andre, B., \& Springael, J. Y. (1994). WWP, a new amino acid motif present in single or multiple copies in various proteins including dystrophin and the SH3-binding Yes-associated protein YAP65. Biochem Biophys Res Commun, 205(2), 1201-1205.

http://dx.doi.org/10.1006/bbrc.1994.2793

Barlow, A. L., Macleod, A., Noppen, S., Sanderson, J., \& Guerin, C. J. (2010). Colocalization analysis in fluorescence micrographs: verification of a more accurate calculation of pearson's correlation coefficient. Microsc Microanal, 16(6), 710-724.

http://dx.doi.org/10.1017/S143192761009389X

Basu, S., Totty, N. F., Irwin, M. S., Sudol, M., \& Downward, J. (2003). Akt phosphorylates the Yes-associated protein, YAP, to induce interaction with 14-3-3 and attenuation of p73-mediated apoptosis. Mol Cell, 11(1), 11-23.

Chan, E. Y., Longatti, A., McKnight, N. C., \& Tooze, S. A. (2009). Kinase-inactivated ULK proteins inhibit autophagy via their conserved C-terminal domains using an Atg13-independent mechanism. Mol Cell Biol, 29(1), 157-171. http://dx.doi.org/10.1128/MCB.01082-08

Chen, H. I., \& Sudol, M. (1995). The WW domain of Yes-associated protein binds a proline-rich ligand that differs from the consensus established for Src homology 3-binding modules. Proc Natl Acad Sci U S A, 92(17), 7819-7823.

Chen, Y., Liu, X. R., Yin, Y. Q., Lee, C. J., Wang, F. T., Liu, H. Q., . . . Liu, J. (2014). Unravelling the multifaceted roles of Atg proteins to improve cancer therapy. Cell Prolif, 47(2), 105-112. http://dx.doi.org/10.1111/cpr.12095

Codogno, P., Ogier-Denis, E., \& Houri, J. J. (1997). Signal transduction pathways in macroautophagy. Cell Signal, 9(2), 125-130.

Dodson, M., Darley-Usmar, V., \& Zhang, J. (2013). Cellular metabolic and autophagic 
pathways: traffic control by redox signaling. Free Radic Biol Med, 63, 207-221.

http://dx.doi.org/10.1016/j.freeradbiomed.2013.05.014

Egan, D. F., Shackelford, D. B., Mihaylova, M. M., Gelino, S., Kohnz, R. A., Mair, W., .. . Shaw, R. J. (2011). Phosphorylation of ULK1 (hATG1) by AMP-activated protein kinase connects energy sensing to mitophagy. Science, 331(6016), 456-461.

http://dx.doi.org/10.1126/science.1196371

Galliot, B. (2006). Autophagy and self-preservation: a step ahead from cell plasticity? Autophagy, 2(3), 231-233.

Huang, J., Wu, S., Barrera, J., Matthews, K., \& Pan, D. (2005). The Hippo signaling pathway coordinately regulates cell proliferation and apoptosis by inactivating Yorkie, the Drosophila Homolog of YAP. Cell, 122(3), 421-434. http://dx.doi.org/10.1016/j.cell.2005.06.007

Huang, Y., Chuang, A. Y., \& Ratovitski, E. A. (2011). Phospho-DeltaNp63alpha/miR-885-3p axis in tumor cell life and cell death upon cisplatin exposure. Cell Cycle, 10(22), 3938-3947. http://dx.doi.org/10.4161/cc.10.22.18107

Jung, C. H., Jun, C. B., Ro, S. H., Kim, Y. M., Otto, N. M., Cao, J., . . Kim, D. H. (2009). ULK-Atg13-FIP200 complexes mediate mTOR signaling to the autophagy machinery. Mol Biol Cell, 20(7), 1992-2003. http://dx.doi.org/10.1091/mbc.E08-12-1249

Kanai, F., Marignani, P. A., Sarbassova, D., Yagi, R., Hall, R. A., Donowitz, M., . . Yaffe, M. B. (2000). TAZ: a novel transcriptional co-activator regulated by interactions with 14-3-3 and PDZ domain proteins. EMBO J, 19(24), 6778-6791.

http://dx.doi.org/10.1093/emboj/19.24.6778

Kelekar, A. (2005). Autophagy. Ann $N \quad Y$ Acad Sci, 1066, 259-271. doi: 10.1196/annals.1363.015

Lieu, K. G., Shim, E. H., Wang, J., Lokareddy, R. K., Tao, T., Cingolani, G., . . Jans, D. A. (2014). The p53-induced factor Ei24 inhibits nuclear import through an importin beta-binding-like domain. J Cell Biol, 205(3), 301-312.

http://dx.doi.org/10.1083/jcb.201304055

McAlpine, F., Williamson, L. E., Tooze, S. A., \& Chan, E. Y. (2013). Regulation of nutrient-sensitive autophagy by uncoordinated 51-like kinases 1 and 2. Autophagy, 9(3), 361-373. http://dx.doi.org/10.4161/auto.23066

Proikas-Cezanne, T., Ruckerbauer, S., Stierhof, Y. D., Berg, C., \& Nordheim, A. (2007). Human WIPI-1 puncta-formation: a novel assay to assess mammalian autophagy. FEBS Lett, 581(18), 3396-3404. http://dx.doi.org/10.1016/j.febslet.2007.06.040

Proikas-Cezanne, T., Waddell, S., Gaugel, A., Frickey, T., Lupas, A., \& Nordheim, A. (2004). WIPI-1alpha (WIPI49), a member of the novel 7-bladed WIPI protein family, is aberrantly expressed in human cancer and is linked to starvation-induced autophagy. Oncogene, 23(58), 9314-9325. http://dx.doi.org/10.1038/sj.onc.1208331 


\section{Macrothink}

Shin, S. H., Lee, E. J., Chun, J., Hyun, S., \& Kang, S. S. (2015). ULK2 Ser 1027 Phosphorylation by PKA Regulates Its Nuclear Localization Occurring through Karyopherin Beta 2 Recognition of a PY-NLS Motif. PLoS One, 10(6), e0127784. http://dx.doi.org/10.1371/journal.pone.0127784

Sudol, M., Bork, P., Einbond, A., Kastury, K., Druck, T., Negrini, M., . . Lehman, D. (1995). Characterization of the mammalian YAP (Yes-associated protein) gene and its role in defining a novel protein module, the WW domain. J Biol Chem, $270(24), 14733-14741$.

Sudol, M., Chen, H. I., Bougeret, C., Einbond, A., \& Bork, P. (1995). Characterization of a novel protein-binding module--the WW domain. FEBS Lett, 369(1), 67-71.

Terman, A., \& Brunk, U. T. (2005). Autophagy in cardiac myocyte homeostasis, aging, and pathology. Cardiovasc Res, 68(3), 355-365. http://dx.doi.org/10.1016/j.cardiores.2005.08.014

Vassilev, A., Kaneko, K. J., Shu, H., Zhao, Y., \& DePamphilis, M. L. (2001). TEAD/TEF transcription factors utilize the activation domain of YAP65, a Src/Yes-associated protein localized in the cytoplasm. Genes Dev, 15(10), 1229-1241.

http://dx.doi.org/10.1101/gad.888601

Wang, K., Degerny, C., Xu, M., \& Yang, X. J. (2009). YAP, TAZ, and Yorkie: a conserved family of signal-responsive transcriptional coregulators in animal development and human disease. Biochem Cell Biol, 87(1), 77-91. http://dx.doi.org/10.1139/O08-114

Yan, J., Kuroyanagi, H., Tomemori, T., Okazaki, N., Asato, K., Matsuda, Y., . . Muramatsu, M. (1999). Mouse ULK2, a novel member of the UNC-51-like protein kinases: unique features of functional domains. Oncogene, 18(43), 5850-5859.

http://dx.doi.org/10.1038/sj.onc.1202988

Zhou, X., Babu, J. R., da Silva, S., Shu, Q., Graef, I. A., Oliver, T., . . . Wang, F. (2007). Unc-51-like kinase 1/2-mediated endocytic processes regulate filopodia extension and branching of sensory axons. Proc Natl Acad Sci U S A, 104(14), 5842-5847.

http://dx.doi.org/10.1073/pnas.0701402104

\section{Copyright Disclaimer}

Copyright for this article is retained by the author(s), with first publication rights granted to the journal.

This is an open-access article distributed under the terms and conditions of the Creative Commons Attribution license (http://creativecommons.org/licenses/by/3.0/). 